\title{
Minimally invasive aortic valve replacement with Perceval S sutureless valve: Early outcomes and one-year survival from two European centers
}

\author{
Antonio Miceli, MD, PhD, ${ }^{\mathrm{a}, \mathrm{b}}$ Giuseppe Santarpino, $\mathrm{MD},{ }^{\mathrm{c}}$ Steffen Pfeiffer, MD, ${ }^{\mathrm{c}}$ Michele Murzi, MD, ${ }^{\mathrm{a}}$ \\ Daniyar Gilmanov, MD, ${ }^{\mathrm{a}}$ Giovanni Concistré, MD, ${ }^{\mathrm{c}}$ Eugenio Quaini, MD, ${ }^{\mathrm{a}}$ Marco Solinas, MD, ${ }^{\mathrm{a}}$ \\ Theodor Fischlein, MD, ${ }^{\mathrm{c}}$ and Mattia Glauber, $\mathrm{MD}^{\mathrm{a}}$
}

\begin{abstract}
Objective: The aim of our study was to evaluate the early outcomes and 1-year survival of patients undergoing minimally invasive aortic valve replacement with the Perceval S sutureless valve for severe aortic stenosis.

Methods: From March 2010 to March 2013, 281 high-risk patients underwent minimally invasive aortic valve replacement with the Perceval S sutureless valve through either right anterior minithoracotomy $(\mathrm{n}=164)$ or upper ministernotomy $(\mathrm{n}=117)$ at 2 cardiac centers.
\end{abstract}

\begin{abstract}
Results: The overall in-hospital mortality was $0.7 \%$ ( 2 patients). The overall median cardiopulmonary bypass and crossclamp time was 81 minutes (interquartile range, 68-98) and 48 minutes (interquartile range, 37-60), respectively. Postoperative stroke occurred in 5 patients $(1.8 \%)$. The incidence of paravalvular leak greater than 1 of 4 and atrioventricular block requiring pacemaker implantation was $1.8 \%$ (5 patients) and $4.2 \%$ (12 patients), respectively. No migration occurred, and the mean postoperative gradient was $13 \pm 4 \mathrm{~mm} \mathrm{Hg}$. At a median follow-up of 8 months (interquartile range, 4-14), the overall survival was $90 \%$.
\end{abstract}

Conclusions: Minimally invasive aortic valve replacement with the Perceval S sutureless valve in high-risk patients is a safe and reproducible procedure associated with excellent hemodynamic results, postoperative outcomes, and 1-year survival. (J Thorac Cardiovasc Surg 2014;148:2838-43)

See related commentary on pages $2843-4$.

The clinical outcomes after elective isolated aortic valve replacement (AVR) have significantly improved in previous years. ${ }^{1}$ According to the recent Fourth European Association for Cardio-Thoracic Surgery adult cardiac surgery database report, the overall operative mortality has ranged from 1.2 to $\leq 14 \%$, depending on patient age and overall risk profile. ${ }^{2}$ Despite these results, less invasive procedures have been developed as an alternative to the conventional technique to reduce the surgical trauma and preserve the same quality, safety, and efficacy of the full sternotomy approach. ${ }^{3}$ Minimally invasive AVR (MIAVR) has shown excellent results in terms of mortality, morbidity, and patient

From the Cardiothoracic Department, ${ }^{\mathrm{a}}$ Fondazione Toscana G. Monasterio, Massa, Italy; Bristol Heart Institute, ${ }^{\mathrm{b}}$ University of Bristol, United Kingdom; and Cardiac Surgery, ${ }^{\mathrm{c}}$ Klinikum Nürnberg, Nürnberg, Germany.

Disclosures: Drs Santarpino, Pfeffer, Fischlein, Solinas, and Glauber disclose a financial relationship with Sorin Group. All other authors have nothing to disclose. Drs A.M. and G.S. contributed equally to the report.

Received for publication Nov 15, 2013; revisions received Feb 6, 2014; accepted for publication Feb 28, 2014; available ahead of print April 1, 2014.

Address for reprints: Antonio Miceli, MD, PhD, Cardiothoracic Department, Fondazione Toscana G. Monasterio, Massa 54100, Italy (E-mail: antoniomiceli79@ alice.it).

0022-5223/\$36.00

Copyright (c) 2014 by The American Association for Thoracic Surgery

http://dx.doi.org/10.1016/j.jtcvs.2014.02.085 satisfaction, providing less pain, faster recovery, and a shorter hospital stay. ${ }^{4-7}$ However, traditionalists have claimed that MIAVR is technically more complex, requires a distinct learning curve, and that the only clear benefit to the patient is cosmesis. ${ }^{8,9}$ Furthermore, the longer crossclamp and cardiopulmonary bypass (CPB) times associated with the MIAVR approach have raised some concerns regarding its safety in elderly and high-risk patients, because they are well-known risk factors for adverse outcomes after cardiac surgery. ${ }^{10,11}$ In this setting, the implantation of sutureless valves might facilitate the MIAVR approach, reducing the operative times.

The Perceval S sutureless valve (Sorin Biomedica Cardio Srl, Salluggia, Italy) is a new self-expanding prosthesis made of bovine pericardium mounted in a nitinol stent, designed as an alternative to the traditional prostheses to simplify surgical implantation. Recently, the Perceval S has shown excellent results in term of postoperative outcomes and hemodynamic performance, decreasing the operative times. ${ }^{12-15}$ The aim of our study was to evaluate the early outcomes and 1-year survival of patients who had undergone MIAVR for severe aortic valve stenosis with the Perceval S sutureless valve at 2 different institutions.

\section{METHODS}

\section{Patient Selection and Data Collection}

A retrospective, observational study was undertaken of prospectively collected data from consecutive patients with severe aortic stenosis 


\section{Abbreviations and Acronyms \\ $\mathrm{AVR}=$ aortic valve replacement \\ $\mathrm{CPB}=$ cardiopulmonary bypass \\ $\mathrm{L} \quad=$ large \\ $\mathrm{M} \quad=$ medium \\ MIAVR $=$ minimally invasive AVR \\ MS = ministernotomy \\ PVL = paravalvular leakage \\ $\mathrm{RT}=$ right anterior minithoracotomy \\ $\mathrm{S} \quad=$ small \\ STS = Society of Thoracic Surgeons \\ TAVI $=$ transcatheter aortic valve implantation}

(mean gradient $>40 \mathrm{~mm} \mathrm{Hg}$ or an aortic valve area $<1 \mathrm{~cm}^{2}$ ) undergoing MIAVR from March 2010 to March 2013. The completed data collection forms were entered in a local databases and included several sections completed by the anesthetists, cardiac surgeons, and perfusionists involved in the care of the patients. A total of 281 consecutive patients with severe aortic stenosis underwent MIAVR with the Perceval S sutureless valve at 2 European cardiac centers (Fondazione Toscana G. Monsaterio, Massa, Italy; and Klinikum Nürnberg, Nürnberg, Germany). Of the 281 patients, 164 underwent right anterior minithoracotomy (RT) and 117 underwent a ministernotomy (MS) approach. The local committee of institutional review boards approved the study to meet ethical and legal requirements, and individual patient consent was waived.

The selection criteria for the Perceval S sutureless valve were severe calcified aortic valve stenosis, or steno-insufficiency, age $\geq 65$ years, EuroSCORE I $>5 \%$, and a small calcified aortic root and/or annulus. The exclusion criteria for valve implantation were acute endocarditis, bicuspid aortic valve replacement with asymmetric sinus of Valsalva, dilatation of the ascending aorta $>4$ $\mathrm{cm}$ in the sinotubular junction, and a ratio between the diameter of the sinus of Valsalva and diameter of the superior annulus $>1.3$ (a ratio $>1.3$ can prevent correct fixation of the valve stent on the aorta). Mortality was defined as any death occurring within 30 days of surgery. Postoperative stroke was diagnosed if evidence was found of a new neurologic deficit with morphologic substrate confirmed by computed tomography or nuclear magnetic resonance imaging. Before discharge, all patients underwent transthoracic echocardiography. The grade of paravalvular leakage (PVL) regurgitation was determined from the color Doppler imaging findings and classified into 4 grades: trivial, 1 of 4; mild, 2 of 4; moderate, 3 of 4; and severe, 4 of 4 . The follow-up data were $100 \%$ complete.

\section{Preoperative Planning and Surgical Procedures}

All patients undergoing MIAVR underwent an accurate preoperative transthoracic echocardiographic study for a better evaluation of the aortic valve, aortic annulus diameter, and symmetry of the Valsalva sinuses. Patients who were a candidate for a RT approach underwent 64-slice thoracic computed tomography without contrast enhancement to evaluate the relationship among the aortic valve, sternum, and intercostal spaces. The patients were considered suitable for a RT approach if, at the level of the main pulmonary artery, the ascending aorta was rightward with respect to the right sternal border and the distance from the ascending aorta to the sternum did not exceed $10 \mathrm{~cm}$. The MS and RT approaches were chosen according to center preferences. Specifically, MS was the main approach in Nuremberg $(95 \%)$, and RT was mainly performed in Massa $(90 \%$, with the remaining not suitable for RT for anatomic reasons).

The surgical techniques have been previously reported. ${ }^{16-18}$ In brief, RT was performed through a 5- to 7-cm skin incision placed at the level of the second intercostal space. Direct aortic cannulation was performed using flexible cannulas, such as Easyflow (Sorin, Sallugia, Italy), and venous drainage was achieved with a Bio-Medicus Multistage cannula (Medtronic, Minneapolis, Minn) or remote access perfusion cannula (Sorin) and inserted through the femoral vein into the right atrium, and the correct position was reached using the Seldinger technique under transesophageal echocardiographic guidance. ${ }^{16}$ The MS sternotomy approach was achieved through a 6- to $10-\mathrm{cm}$ midline vertical skin incision, performing a partial J sternotomy at the third to fifth intercostal space or a V-shaped ministernotomy at the level of the second intercostal space. ${ }^{17,18}$ After CPB had been established, a left ventricular vent was placed through the right superior pulmonary vein, and the patients were cooled to $34^{\circ} \mathrm{C}$. The ascending aorta was clamped with the DeBakey crossclamp or with the Glauber clamp (Cardiomedical $\mathrm{GmbH}$, Langenhagen, Germany, distributed by Sorin), and antegrade cardioplegic solution was given into the aortic root or selectively into the coronary ostia using warm blood cardioplegia. In all cases, the surgical field was flooded with carbon dioxide until closure of the aortotomy.

\section{Perceval S Implantation}

The transverse aortotomy was performed approximately 1.5 to $2 \mathrm{~cm}$ higher than ordinary aortotomy for AVR. The reference point was the inferior margin of the Concato preaortic bundle. The diseased native valve was completely removed and the aortic annulus thoroughly decalcified and sized. The valve sizer was designed so that the intra-annular head of the sizer (yellow) has the same external diameter as the supra-annular head (white) of the smaller size. For the appropriate sizing, the native annulus should allow the passage of the intra-annular head but not the supra-annular head of the same sizer (eg, intra-annular head of size large [L] should correspond to the supraannular head of size medium [M]). Afterward, 3 guiding 4-0 Prolene sutures were placed at the nadir point of each valve sinuses to act as a reference for accurate alignment of the inflow portion of the prosthesis into the aortic annulus. The valve was collapsed using a specific device system and connected to the guiding sutures through 3 bottom holes placed on the midpart of the inflow ring. The deployment system was parachuted down into the aortic root and the valve released into the aortic annulus. The inflow ring should completely cover the aortic annulus, such that no part of the native aortic annulus is exposed. Although not recommended by Sorin, it is still possible to adjust the valve position using the forceps. Once coaptation of the 3 leaflets had been checked, a balloon was inserted into the sutureless valve and expanded with warm saline solution for 30 seconds at a pressure of $4 \mathrm{mBar}$. Finally, the 3 guiding sutures were removed; the valve was again checked for the correct position, and the aortotomy was closed using 4-0 or 5-0 running sutures. The patient was weaned from $\mathrm{CPB}$, and the valve was evaluated for the presence of PVL using transesophageal echocardiography.

Currently, 3 sizes are available: small $(\mathrm{S})(21 \mathrm{~mm}), \mathrm{M}(23 \mathrm{~mm})$, and $\mathrm{L}(25 \mathrm{~mm})$. The extra large (XL) size is still under investigation in a multicenter study for the European Commission ("Conformite Europeene") approval and was used exclusively by the Nuremberg group.

\section{Statistical Analysis}

Continuous data are presented as the mean \pm standard deviation or median and interquartile range (IQR) and categorical data as percentages. The Kolmogorov-Smirnov test was used to check for normality of the postoperative gradients before additional analysis. Differences between mean postoperative gradients and valve sizes were tested using the KruskalWallis test. Kaplan-Meier estimates were used to determine total survival and event-free survival (defined as the absence of reoperation, endocarditis, stroke, and death). Statistical analysis was performed using the Statistical Package for Social Sciences, version 15.0 (SPSS, Chicago, Ill).

\section{RESULTS}

The baseline and intraoperative characteristics are reported in Tables 1 and 2. All valves were successfully 
TABLE 1. Preoperative patient characteristics $(n=281)$

\begin{tabular}{lc}
\hline \multicolumn{1}{c}{ Characteristic } & Value \\
\hline Age (y) & $76.8 \pm 6$ \\
Female gender & $174(61.9)$ \\
Diabetes & $62(22.1)$ \\
Chronic pulmonary disease & $45(16)$ \\
Hypertension & $238(84.7)$ \\
NYHA III-IV & $134(47.7)$ \\
Extracardiac arterioplasty & $33(11.7)$ \\
Previous cardiac operations & $5(1.8)$ \\
Preoperative EF $(\%)$ & $56.8 \pm 8.5$ \\
Poor EF $(<30 \%)$ & $7(2.5)$ \\
Chronic renal dysfunction $(\mathrm{SCr}>2 \mathrm{mg} / \mathrm{dL})$ & $11(3.9)$ \\
Pulmonary hypertension & $37(13.2)$ \\
Logistic EuroSCORE & $8(5-12)$ \\
RT approach & $164(58.4)$ \\
MS approach & $117(41.6)$ \\
\hline
\end{tabular}

Data presented as mean \pm standard deviation, $\mathrm{n}(\%)$, or median (interquartile range). NYHA, New York Heart Association; $E F$, ejection fraction; $S C r$, serum creatinine; $R T$, right anterior minithoracotomy; $M S$, ministernotomy.

implanted using either RT or MS. The median CPB and crossclamp time was 81 minutes (IQR, 68-98) and 48 minutes (IQR, 37-60), respectively. In the RT group, the CPB and crossclamp time was 74 minutes (IQR 87-107) and 55 minutes (IQR 47-65), respectively. In the MS group, the CPB and crossclamp time was 72 minutes (IQR 58-89) and 37 minutes (IQR 30-46; Table 2). The most common size used in both approaches was size $\mathrm{L}$ $(\mathrm{n}=141,50.2 \%)$. Of the patients, $34(12.1 \%)$ received size $\mathrm{S}$ and 97 (34.5\%) size M. The XL size was implanted in 9 patients $(3.2 \%)$ through the MS approach in Nuremberg as a part of the multicenter study for the European Commission approval.

Overall, in-hospital mortality was $0.7 \%$ ( 1 patient died of multiorgan failure and 1 of liver insufficiency), with a predicted median EuroSCORE of $8 \%$ (IQR, 5\%-12\%). The outcomes are reported in Table 3. Four patients $(1.4 \%)$ required conversion to full sternotomy because of bleeding. No valve migration was reported. In 5 patients, owing to incorrect alignment of the prosthesis into the aortic annulus, the valve was explanted, recollapsed, and reimplanted with success. Two patients required better

TABLE 2. Intraoperative characteristics

\begin{tabular}{lccc}
\hline \multicolumn{1}{c}{ Characteristic } & MIAVR $(\mathbf{n}=\mathbf{2 8 1})$ & RT $(\mathbf{n}=\mathbf{1 6 4})$ & MS $(\mathbf{n}=\mathbf{1 1 7})$ \\
\hline CPB time (min) & $81(68-98)$ & $74(87-107)$ & $72(58-89)$ \\
Crossclamp time (min) & $48(37-60)$ & $55(47-65)$ & $37(30-46)$ \\
Perceval S size & & & \\
$\quad$ S $(21 \mathrm{~mm})$ & $34(12.1)$ & $21(12.8)$ & $13(11.1)$ \\
M $(23 \mathrm{~mm})$ & $97(34.5)$ & $57(34.8)$ & $40(34.2)$ \\
L $(25 \mathrm{~mm})$ & $141(50.2)$ & $86(52.4)$ & $55(47)$ \\
XL $(27 \mathrm{~mm})$ & $9(3.2)$ & 0 & $9(7.7)$ \\
\hline
\end{tabular}

Data presented as median (interquartile range) or $\mathrm{n}(\%)$. MIAVR, Minimally invasive aortic valve replacement; $R T$, right anterior minithoracotomy; $M S$, ministernotomy; $C P B$, cardiopulmonary bypass; $S$, small; $M$, medium; $L$, large; $X L$, extra large.
TABLE 3. Postoperative outcomes $(n=281)$

\begin{tabular}{lc}
\hline \multicolumn{1}{c}{ Outcomes } & Value \\
\hline Mortality & $2(0.7)$ \\
Stroke & $5(1.8)$ \\
Re-exploration for bleeding & $8(2.8)$ \\
Conversion to sternotomy & $4(1.4)$ \\
AV block requiring PMK & $12(4.2)$ \\
PVL $>1$ of 4 & $5(1.8)$ \\
ICU stay (d) & $1(1-2)$ \\
Ward stay (d) & $8(6-10)$ \\
\hline
\end{tabular}

Data presented as $\mathrm{n}(\%)$ or median (interquartile range). $A V$, Atrioventricular; $P M K$, pacemaker; $I C U$, intensive care unit; $P V L$, paravalvular leakage.

annulus decalcification. These complications occurred in the first phase of our study, suggesting a possible learning curve effect. Postoperative stroke occurred in 5 patients $(1.8 \%)$. Twelve patients $(6 \%)$ had trivial (1 of 4$)$ PVL. The incidence of PVL $>1$ of 4 was $1.8 \%$ (5 patients); 4 patients $(1.4 \%$ ) had mild ( 2 of 4 ) PVL, with 2 diagnosed at discharge and 2 in the operating room. One patient $(0.3 \%)$ had mild (2 of 4) PVL in the operating room that had become moderate (3 of 4 ) at discharge. In these patients, we chose to leave the PVL owing to the patients' age and comorbidities. The incidence of atrioventricular block requiring pacemaker implantation was 4.2\% (12 patients). At discharge, the mean and peak postoperative gradient was $13 \pm 4$ and $24 \pm 8 \mathrm{~mm} \mathrm{Hg}$, respectively. No difference was found between the valve size and the mean gradient. The mean postoperative gradient for each size was $13.5 \pm 5.1 \mathrm{~mm} \mathrm{Hg}$ for size $\mathrm{S}$, $13.7 \pm 4.5 \mathrm{~mm} \mathrm{Hg}$ for size $\mathrm{M}, 12.8 \pm 4 \mathrm{~mm} \mathrm{Hg}$ for size $\mathrm{L}$, and $13.5 \pm 3.2 \mathrm{~mm} \mathrm{Hg}$ for size $\mathrm{XL}(P=.36)$.

At a median follow-up of 8 months (IQR, 4-14), the 1 -year survival was $90 \%$ (Figure 1). Overall, the event-free survival was $82 \%$. Finally, 1 patient required reoperation for endocarditis caused by Aspergillus spp, and the freedom from reoperation or endocarditis was $99.6 \%$.

\section{DISCUSSION}

Our study has shown that minimally invasive aortic valve procedures using the Perceval S sutureless valve with an RT or MS approach is a safe procedure associated with a low incidence of mortality and postoperative complications. Despite the increased risk profile and advanced age of our patients, the in-hospital mortality was $0.7 \%$, lower than the recent mortality rate reported in The Society of Thoracic Surgeons (STS) and European Association for Cardio-Thoracic Surgery adult cardiac surgery database. Moreover, the low incidence of PVL and permanent pacemaker implantation and the short operative times have confirmed the safety and feasibility of this valve in minimally invasive approaches, making this access technically easier and more reproducible. Finally, we have 


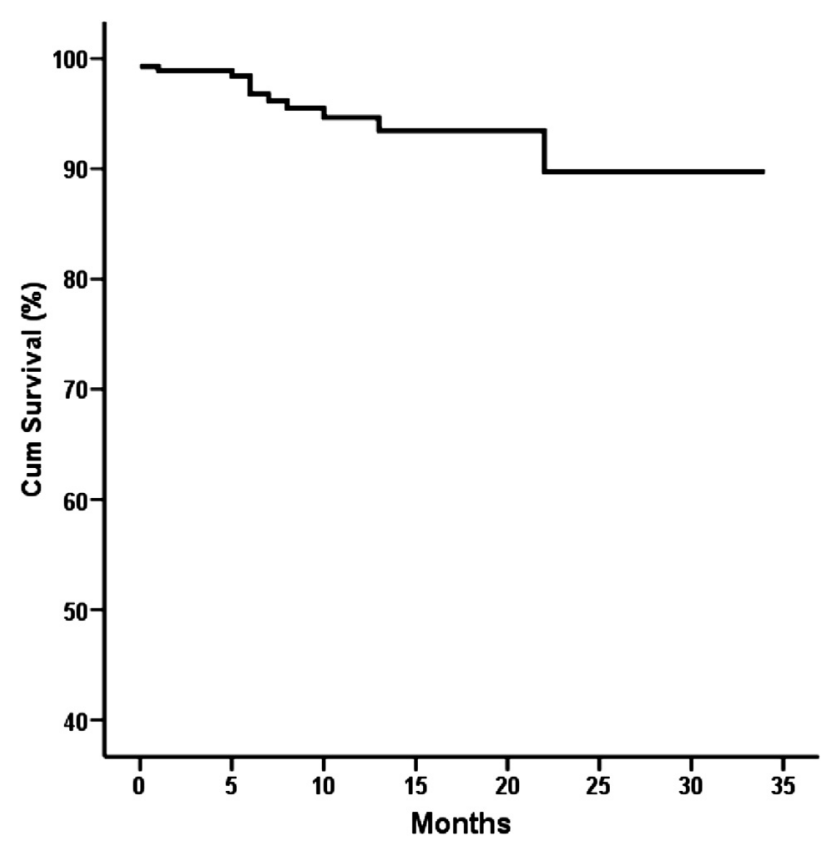

FIGURE 1. Graph showing cumulative survival.

demonstrated excellent hemodynamic performance, regardless of the prosthesis size. Compared with standard AVR, MIAVR has shown excellent outcomes in terms of postoperative complications and hospital stay, ${ }^{4,5}$ suggesting it might be of benefit particularly in older and fragile patients. ${ }^{3-6}$ Despite these results, controversies exist regarding the minimally invasive approach, because several prospective randomized trials have failed to demonstrate any theoretical advantage. ${ }^{4}$ Furthermore, it has been shown that MIAVR is associated with a longer $\mathrm{CPB}$ and aortic crossclamp times compared with traditional surgery. ${ }^{4,5}$ The reduction in working space for the exposure and implantation of the prosthetic valves is more complex and technically challenging (especially in small aortic annuli), reflecting the longer operative times and the slow learning curve. That has raised some concerns regarding its safety in high-risk patients, because the longer CPB and crossclamp times might eliminate the benefits offered by the minimally invasive approach. ${ }^{8,9}$ Recently, we have described a low rate of complication after MS or RT; however, we have confirmed the longer operative times compared with the full sternotomy. ${ }^{7,19}$ The drawback of the increasing surgical times could be avoided by the adoption of sutureless technology, which might facilitate the MIAVR approach.

As an alternative to the biologic stented valve, the Perceval S sutureless valve was designed to avoid passing the stitches through the annulus and suture knotting to minimize the surgical trauma to the aortic annulus and, consequently, reduce the ischemic time. To date, several trials have demonstrated excellent clinical and hemodynamic outcomes for patients undergoing AVR with the
Perceval S. ${ }^{12-15}$ However, these studies had focused mainly on full sternotomy. Few studies have focused on a minimally invasive approach. ${ }^{15,17,20}$ In a series of 32 high-risk patients, Flameng and colleagues ${ }^{12}$ implanted Perceval $\mathrm{S}$ valves within 20 minutes of aortic crossclamping and reported no operative mortality and excellent clinical and hemodynamic outcomes. However, only 1 patient had undergone a partial upper ministernotomy. In a larger study, Folliguet and colleagues ${ }^{13}$ reported presented the outcomes of 208 high-risk patients undergoing AVR. The mean crossclamp time for isolated AVR was 33 minutes, with an overall in-hospital mortality of $2.4 \%$. Survival at 12 months was $87 \%$, and freedom from reoperation was $96 \%$. Although the investigators had concluded that the Perceval $\mathrm{S}$ can be easily implanted using MIAVR, only $22 \%$ of the patients $(n=45)$ had undergone a mini-AVR approach. ${ }^{13}$ Nevertheless, no clinical outcomes associated with MIAVR were reported. Finally, Santarpino and colleagues ${ }^{14}$ confirmed these results, including 51 patients who had undergone J ministernotomy. In contrast, Shrestha and colleagues ${ }^{15}$ described the outcomes of 35 patients who had undergone ministernotomy and concluded that the Perceval S sutureless valve is a technically more reproducible alternative for mini-AVR, even in small and calcified aortic roots. To the best of our knowledge, our study is the largest reporting the outcomes of patients undergoing MIAVR. The strength of our report was that we have described the postoperative results of the 2 most common minimally invasive approaches. The in-hospital mortality was $0.7 \%$, and, despite their high-risk profile and advanced age, the morbidity was low, and all the patients recovered well from the surgery. Moreover, compared with our previous studies of MIAVR with stented valves, we found a $38 \%$ and $40 \%$ reduction in the crossclamp and CPB time in the RT group and $43 \%$ and $35 \%$ in the MS group, confirming that sutureless valves can facilitate the surgical procedure, respectively. Specifically, the mean crossclamp and CPB time for RT and MS using stented valves was $89 \pm 32$ and $123 \pm 45$ minutes in the RT group and $66 \pm 20$ and $105 \pm 35$ minutes in the MS group. ${ }^{16,21} \mathrm{We}$ recognize that our operative times were still longer than those reported by other Perceval studies; however, those studies focused mainly on median standard sternotomy or reported a low percentage of patients undergoing MIAVR. Furthermore, the STS national database reported that the mean crossclamp and CPB time for isolated AVR with full sternotomy was 78 and 106 minutes, respectively (available at: www.sts.org/documents/pdf/Spring2005STS ExecutiveSummary.pdf).

Only 1 patient required reoperation after 6 months for endocarditis. Because the aortic root was intact, we decided to implant a new Perceval S using an RT approach. Finally, the 1-year survival was $90 \%$, in line with the recent data reported by the STS database. ${ }^{22}$ Because of our outstanding 
postoperative outcomes, we believe that the MIAVR approach associated with sutureless valves might be considered an alternative procedure to transcatheter aortic valve implantation (TAVI) procedures for high-risk patients. Compared with surgery, transcatheter implantation has shown similar results in terms of mortality but with an increased risk of PVL and stroke. ${ }^{23}$ Although TAVI represents the real MIAVR because it is performed without $\mathrm{CPB}$, the main limitation of TAVI is that the native stenotic valve is left in place, resulting in a high degree of stroke, PVL, and atrioventricular block. The surgery has the advantage of removing the diseased valve, resulting in a lower incidence of PVL and stroke. ${ }^{23,24}$ It has been shown that PVL has a negative effect on survival. ${ }^{23}$ In our experience, the incidence of postoperative PVL $>1$ of was $1.8 \%$, much lower than that for those undergoing TAVI $(12.2 \%){ }^{23}$ Furthermore, this rate was even lower than that from other Perceval S reports $(4 \%-15 \%))^{12-15}$ In this regard, the Sorin company has recommended removing only eccentric and bulky intra-annular calcifications. In contrast, we strongly believe that accurate and complete decalcification of the aortic annulus will further reduce the incidence of paravalvular aortic regurgitation. Finally, in our series, the rate of postoperative stroke was similar to that reported by the STS database, confirming the safety of this valve compared with other valves. ${ }^{1}$

Finally, an economic comment should be made. A cost-utility analysis of TAVI in Belgium concluded that it is not recommended to reimburse for TAVI for high-risk patients because the patients had no survival benefit after 1 year, the risk of stroke was double, and the costs were significantly higher. ${ }^{25}$ In contrast, an economic model has shown that compared with stented valves the use of the Perceval $\mathrm{S}$ valve might be associated with fewer complications and with savings, mainly related to a reduction in the surgery costs and intensive care unit and hospital bed days. ${ }^{26}$ Although these valves are more expensive, the combination of MIAVR and sutureless technology might minimize the risk of postoperative complications and, therefore, reduce hospital costs. A well-designed study is required to evaluate the cost/benefit of TAVI procedures versus MIAVR using sutureless valves. Also, prospective randomized trials are required to confirm our suppositions.

\section{Study Limitations}

Our study was a retrospective analysis of 2 institutional, observational, prospectively collected databases. Our study included patients undergoing 2 different approaches that cannot be directly comparable, because RT requires some anatomic conditions that are not required for MS. Moreover, RT was mainly performed in Massa and MS in Nuremberg. Although surprising, we found similar gradients among the valve sizes at discharge. According to the recommendations of the American Society of
Echocardiography, the blood velocity across a prosthetic valve will be dependent on several factors, including the valve size and type and flow. The flow rate will be determined by the cardiac output, ventricular function, viscosity, left ventricular outflow, and body surface area. These factors could have affected the mean gradients across the valves. ${ }^{27}$ However, our database did not include this information, and the lack of these data did not allow us to study this topic in depth. Furthermore, no data were available regarding the gradients during the follow-up period. A well-designed prospective study is required to evaluate the potential differences between the 2 minimally invasive approaches and the Perceval $\mathrm{S}$ hemodynamic performances.

\section{CONCLUSIONS}

MIAVR with the Perceval S sutureless valve is a safe and reproducible procedure associated with excellent hemodynamic results, postoperative outcomes, and 1-year survival. Because of these outstanding results, we believe that MIAVR with sutureless valves could be the real alternative to the TAVI procedure for high-risk patients. Prospective randomized trials are required to confirm our hypothesis.

\section{References}

1. Brown JM, O’Brien SM, Wu C, Sikora JA, Griffith BP, Gammie JS. Isolated aortic valve replacement in North America comprising 108,687 patients in 10 years: changes in risks, valve types, and outcomes in the Society of Thoracic Surgeons national database. J Thorac Cardiovasc Surg. 2009;137: 82-90.

2. Bridgewater B, Gummert J, Kinsman R, Walton P. Towards Global Benchmarking: The Fourth EACTS Adult Cardiac Surgical Database Report. Oxfordshire, United Kingdom: Dendrite Clinical Systems Ltd; 2010.

3. Schmitto JD, Mokashi SA, Cohn LH. Minimally-invasive valve surgery J Am Coll Cardiol. 2010;56:455-62.

4. Murtuza B, Pepper JR, Stanbridge RD, Jones C, Rao C, Darzi A, et al Minimally access aortic valve replacement: is it worth it? Ann Thorac Surg 2008;85:1121-31.

5. Brown ML, McKellar SH, Sundt TM, Schaff HV. Ministernotomy versus conventional sternotomy for aortic valve replacement: a systematic review and meta-analysis. J Thorac Cardiovasc Surg. 2009;137:670-9.

6. Tabata M, Umakanthan R, Cohn LH, Bolman RM III, Shekar PS, Chen FY, et al. Early and late outcomes of 1000 minimally invasive aortic valve operations. Eur J Cardiothorac Surg. 2008;33:537-41.

7. Glauber M, Miceli A, Gilmanov D, Ferrarini M, Bevilacqua S, Farneti PA, et al Right anterior minithoracotomy versus conventional aortic valve replacement: a propensity score matched study. J Thorac Cardiovasc Surg. 2013;145:1222-6.

8. Cooley DA. Minimally invasive valve surgery versus the conventional approach. Ann Thorac Surg. 1998;66:1101-5.

9. Foghsgaard S, Schmidt TA, Kjaergard HK. Minimally invasive aortic valve replacement: late conversion to full sternotomy doubles operative time. Tex Heart Inst J. 2009;36:293-7.

10. Al-Sarraf N, Thalib L, Houlihan M, Tolan M, Young V, McGovern E. Cross-clamp time is an independent predictor of mortality and morbidity in low-and high-risk cardiac patients. Int J Surg. 2011;9:104-9.

11. Ranucci M, Frigiola A, Menicanti L, Castelvecchio S, de Vincentiis C, Pistuddi V. Aortic cross-clamp time, new prostheses, and outcome in aortic valve replacement. J Heart Valve Dis. 2012;21:732-9.

12. Flameng W, Herregods MC, Hermans H, Van der Mieren G, Vercalsteren M, Poortmans G, et al. Effect of sutureless implantation of the Perceval S aortic valve bioprosthesis on intraoperative and early postoperative outcomes. J Thorac Cardiovasc Surg. 2011;142:1453-7. 
13. Folliguet TA, Laborde F, Zannis K, Ghorayeb G, Haverich A, Shrestha M. Sutureless Perceval aortic valve replacement: results of two European centers. Ann Thorac Surg. 2012;93:1483-8.

14. Santarpino G, Pfeiffer S, Schmidt J, Concistrè G, Fischlein T. Sutureless aortic valve replacement: first-year single center experience. Ann Thorac Surg. 2012; 94:504-9.

15. Shrestha M, Timm R, Höffeler K, Koigeldiyev N, Khaladj N, Hagl C, et al. Minimally invasive aortic valve replacement with self-anchoring Perceval valve. J Heart Valve Dis. 2013;22:230-5.

16. Glauber M, Miceli A, Bevilacqua S, Farneti PA. Minimally invasive aortic valve replacement via right anterior minithoracotomy: early outcomes and midterm follow-up. J Thorac Cardiovasc Surg. 2011;142:1577-9.

17. Santarpino G, Pfeiffer S, Concistrè G, Fischlein T. Perceval S aortic valve implantation in mini-invasive surgery: the simple sutureless solution. Interact Cardiovasc Thorac Surg. 2012;15:357-60.

18. Karimov JH, Santarelli F, Murzi M, Glauber M. A technique of an upper V-type ministernotomy in the second intercostal space. Interact Cardiovasc Thorac Surg. 2009;9:1021-2.

19. Miceli A, Murzi M, Gilmanov D, Fugà R, Ferrarini M, Solinas M, et al. Minimally invasive aortic valve replacement using right minithoracotomy is associated with better outcomes than ministernotomy. J Thorac Cardiovasc Surg. Epub 2013 Sep 12.

20. Gilmanov D, Farneti PA, Miceli A, Bevilacqua S, Glauber M. Perceval S sutureless aortic valve prosthesis implantation via a right anterior minithoracotomy. Multimedia Manual Cardiothorac Surg. Epub 2013 Jul 12; doi:10.1093/ mmcts/mmt012.

21. Santarpino G, Pfeiffer S, Concistrè G, Grossmann I, Hinzmann M, Fischlein T. The Perceval S aortic valve has the potential of shortening surgical time: does it also result in improved outcomes? Ann Thorac Surg. 2013;96:77-82.

22. Brennan JM, Edwards FH, Zhao Y, O'Brien SM, Douglas PS, Peterson ED, Developing Evidence to Inform Decision About Effectiveness-Aortic Valve Replacement (DEcIDE AVR) Research Team. Long-term survival after aortic valve replacement among high-risk elderly patients in the United States. Insight from The Society of Thoracic Surgeons adult cardiac surgery database, 1991 to 2007. Circulation. 2012;126:1621-9.

23. Miller DC, Blackstone EH, Mack MJ, Svensson LG, Kodali SK, Kapadia S, et al. Transcatheter (TAVR) versus surgical (AVR) aortic valve replacement: occurrence, hazard, risk factors, and consequences of neurological events in the PARTNER trial. J Thorac Cardiovasc Surg. 2012;143:832-43.

24. Neyt M, Van Brabandt H, Devriese S, Van De Sande S. A cost-utility analysis of transcatheter aortic valve implantation in Belgium: focusing on a well-defined and identifiable population. BMJ Open. 2012;2:e001032.

25. D’Onofrio A, Mesina A, Lorusso R, Alfieri OR, Fusari M, Rubino P, et al. Sutureless aortic valve replacement as an alternative treatment for patient belonging to the "gray zone" between transcatheter aortic valve implantation and conventional surgery: a propensity-matched, multicenter analysis. $J$ Thorac Cardiovasc Surg. 2012:144:1010-8.

26. Pradelli L, Zaniolo O. Perceval Sutureless valves in isolated and concomitan AVR procedures: an economic model shows overall decrease of costs for isolated or combined operations. Farmeconomia. Health Economics and Therapeutic Pathways. 2012;13:159-74.

27. Zoghbi WA, Chambers JB, Dusmenil JG, Foster E, Gottdiener JS, Grayburn PA, et al. Recommendations for evaluation of prosthetic valves with echocardiography and Doppler ultrasound: a report From the American Society of Echocardiography's Guidelines and Standards Committee and the Task Force on Prosthetic Valves, developed in conjunction with the American College of Cardiology Cardiovascular Imaging Committee, Cardiac Imaging Committee of the American Heart Association, the European Association of Echocardiography, a registered branch of the European Society of Cardiology, the Japanese Society of Echocardiography and the Canadian Society of Echocardiography, endorsed by the American College of Cardiology Foundation, American Heart Association, European Association of Echocardiography, a registered branch of the European Society of Cardiology, the Japanese Society of Echocardiography, and Canadian Society of Echocardiography. J Am Soc Echocardiogr. 2009;22:975-1014.

\title{
EDITORIAL COMMENTARY
}

\section{Minimally invasive Perceval S implantation: Does this fill a sizable niche between sutured and transcatheter aortic valve replacement?}

\author{
Michael O. Kayatta, MD, Hanna A. Jensen, MD, PhD, and Vinod H. Thourani, MD
}

See related article on pages $2838-43$

\footnotetext{
From the Structural Heart and Valve Center, Division of Cardiothoracic Surgery, Joseph B. Whitehead Department of Surgery, Emory University School of Medicine, Atlanta, Ga.

Disclosures: V.H.T. serves on the advisory boards of St Jude Medical, Edwards Lifesciences, Boston Scientific, and Abbot Medical and engages in research for Medtronic, Edwards Lifesciences, St Jude, and Sorin Medical. All other authors have nothing to disclose with regard to commercial support.

Received for publication Oct 2, 2014; accepted for publication Oct 2, 2014

Address for reprints: Vinod H. Thourani, MD, Emory University Hospital Midtown, 550 Peachtree St NE, 6th Floor, Medical Office Tower, Atlanta, GA 30308 (E-mail: vthoura@emory.edu).

J Thorac Cardiovasc Surg 2014;148:2843-4

$0022-5223 / \$ 36.00$

Copyright $\odot 2014$ by The American Association for Thoracic Surgery

http://dx.doi.org/10.1016/j.jtcvs.2014.10.004
}

In this issue of the Journal, Miceli and colleagues ${ }^{1}$ present a well-written article on their early experience with placement of the sutureless Perceval S (Sorin SpA, Milan, Italy) valve through minimally invasive surgical approaches of either partial sternotomy or right anterior thoracotomy. Of all patients coming to their centers for isolated aortic valve replacement (AVR), 40\% $(\mathrm{n}=281)$ were found eligible for these techniques. These patients were elderly (mean age, 76 years) but on average were a relatively healthy intermediate-risk population. In-hospital outcomes were excellent, with very low mortality and only $1.8 \%$ of patients having greater than trace paravalvular leak. This rate of leaks is extremely low, and has not been seen by other groups. ${ }^{2,3}$ Overall, the new postoperative pacemaker rate was only $4.2 \%$. These results are to be commended and it is to be hoped will be reproducible 